\title{
Stereoscopic vision display technology in real-time three-dimensional echocardiography-guided intracardiac beating-heart surgery
}

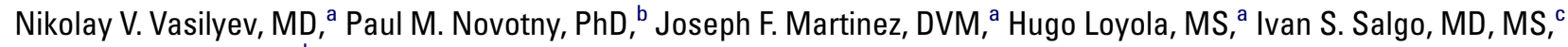
Robert D. Howe, PhD, ${ }^{\mathrm{b}}$ and Pedro J. del Nido, MD ${ }^{\mathrm{a}}$

From the Department of Cardiac Surgery, Children's Hospital Boston, Harvard Medical School, ${ }^{a}$ Boston, Mass; Division of Engineering and Applied Sciences, Harvard University, ${ }^{\mathrm{b}}$ Cambridge, Mass; and Ultrasound Division, Philips Medical Systems, ${ }^{c}$ Andover, Mass.

This work was supported in part by National Institute of Health Grants No. HL-073647 and HL-71128 (Dr del Nido). Ivan Salgo is employed by Philips Healthcare.

Received for publication June 26, 2007; revisions received Nov 16, 2007; accepted for publication Dec 6, 2007.

Address for reprints: Pedro J. del Nido, MD, Department of Cardiac Surgery, Children's Hospital Boston, Harvard Medical School, 300 Longwood Avenue, Boston, MA 02115 (E-mail: pedro.delnido@tch.harvard.edu).

J Thorac Cardiovasc Surg 2008;135:133441

\section{$0022-5223 / \$ 34.00$}

Copyright $\odot 2008$ by The American Association for Thoracic Surgery

doi:10.1016/j.jtcvs.2007.12.045
Objective: Stereoscopic vision display technology has been shown to be a useful tool in image-guided surgical interventions. However, the concept has not been applied to 3-dimensional echocardiography-guided cardiac procedures. We evaluated stereoscopic vision display as an aid for intracardiac navigation during 3-dimensional echocardiography-guided beating-heart surgery in a model of atrial septal defect closure.

Methods: An atrial septal defect $(6 \mathrm{~mm})$ was created in 6 pigs using 3-dimensional echocardiography guidance. The defect was then closed using a catheter-based patch delivery system, and the patch was attached with tissue mini-anchors. Stereoscopic vision was generated with a high-performance volume renderer with stereoscopic glasses. Three-dimensional echocardiography with stereoscopic vision display was compared with 3-dimensional echocardiography with standard display for guidance of surgical repair. Task performance measures for each anchor placement $(\mathrm{N}=32$ per group) were completion time, trajectory of the tip of the anchor deployment device, and accuracy of the anchor placement.

Results: The mean time of the anchor deployment for stereoscopic vision display group was shorter by $44 \%$ compared with the standard display group: $9.7 \pm 0.9 \mathrm{sec}-$ onds versus $17.2 \pm 0.9$ seconds $(P<.001)$. Trajectory tracking of the anchor deployment device tip demonstrated greater navigational accuracy measured by trajectory deviation: $3.8 \pm 0.7 \mathrm{~mm}$ versus $6.1 \pm 0.3 \mathrm{~mm}, 38 \%$ improvement $(P<.01)$. Accuracy of anchor placement was not significantly different: $2.3 \pm 0.3 \mathrm{~mm}$ for the stereoscopic vision display group versus $2.3 \pm 0.3 \mathrm{~mm}$ for the standard display group.

Conclusion: Stereoscopic vision display combined with 3-dimensional echocardiography improved the visualization of 3-dimensional echocardiography ultrasound images, decreased the time required for surgical task completion, and increased the precision of instrument navigation, potentially improving the safety of beating-heart intracardiac surgical interventions.

$\mathrm{T}$ echniques for intracardiac reconstructive surgery in the beating heart offer the promise of avoiding cardiopulmonary bypass while still achieving full repair. The development of reliable imaging tools has been one of the fundamental obstacles to the progress of intracardiac beating-heart surgery. To accomplish the operation safely, the operator has to visualize and manipulate rapidly moving delicate anatomic structures inside a beating heart, in the presence of blood, relying on visual feedback. Real-time 3-dimensional echocardiography (RT3DE) has been shown to be a viable imaging tool for guiding such interventions. ${ }^{1,2}$ RT3DE systems provide ample intraoperative assessment of intracardiac anatomy and enable navigation of surgical instruments toward the target inside the beating heart. To improve the safety of this approach, some technologic advances are needed. In current systems, acquired 3-dimensional (3D) volume data are projected on a conventional 2-dimensional (2D) display where the depth of field is rendered by varying shades of gray. Therefore, 


$$
\begin{aligned}
& \text { Abbreviations and Acronyms } \\
& \begin{aligned}
\text { 2D } & =\text { 2-dimensional } \\
\text { 3D } & =3 \text {-dimensional } \\
\text { 3DE } & =3 \text {-dimensional echocardiography } \\
\text { 3DUS } & =3 \text {-dimensional ultrasound } \\
\text { ASD } & =\text { atrial septal defect } \\
\text { RT3DE } & =\text { real-time 3-dimensional echocardiography } \\
\text { SV } & =\text { stereoscopic vision }
\end{aligned}
\end{aligned}
$$

while operating under RT3DE guidance, the surgeon may not have an adequate display of intracardiac structures in $3 \mathrm{D}$ space and must rely on indirect evidence for depth perception and position of the instruments within the heart.

Recent advances in computer graphics technology have enabled processing of large volumes of 3D data in real time. To use this technology and to take full advantage of 3D ultrasound (3DUS) data for guiding surgery, pre-volume rendered data were streamed to an external computer for volume rendering. Volumetric data sets were then rendered in real time to generate offset images on a stereoscopic vision (SV) display. The purpose of this study was to determine whether the custom-built SV display improved performance during RT3DE-guided beating-heart surgery in a model of atrial septal defect (ASD) creation and repair.

\section{Materials and Methods}

\section{Stereoscopic Vision Display Technology}

Rendering algorithm. To allow real-time stereoscopic visualization, the system must render $30 \mathrm{MB}$ of data every second. This was accomplished by harnessing the computation power of consumerlevel graphics processing units. ${ }^{3,4}$ The fundamental advantage of programmable graphics processing units is their ability to execute highly parallelized routines (shaders). Our implementation uses shaders to cast rays through the volumetric data set in a ray-per-pixel fashion. The intensity $\left(I_{\text {buffer }}\right)$ and opacity $\left(\alpha_{\text {buffer }}\right)$ are compounded by sampling the volumetric data set along the projection ray as equations 1 and 2:

$$
\begin{gathered}
I_{\text {buffer }}=I_{\text {buffer }}+\left(1-\alpha_{\text {buffer }}\right) \alpha_{\text {sample }} I_{\text {sample }} \\
\alpha_{\text {buffer }}=\alpha_{\text {buffer }}+\left(1-\alpha_{\text {buffer }}\right) \alpha_{\text {sample }}
\end{gathered}
$$

The renderer was implemented in DirectX 9.0c using the Pixel Shader 3.0 API on a GeForce FX 7800 (nVidia Corp, Santa Clara, Calif) with 256 MB RAM. The support of hardware loops allows for implementation of the sampling process in a single rendering pass. When rendering typical 3DUS volumetric data sets of size $128 \times 48 \times 204$ in full-screen mode $(640 \times 480$ screen resolution $)$, the renderer maintains highly interactive frame rates of 70 frames per second and above, which provides real-time stereoscopic imaging.

System. RT3DE data were obtained using the X4 matrix transducer on a SONOS 7500 system (Philips Medical Systems, Andover, Mass). The streaming volumes, typically $128 \times 48 \times 204$ voxels, were produced at $25 \mathrm{~Hz}$ and sent over a transmission control proto-
col/Internet protocol network to a personal computer running the rendering algorithm described above. As the data were received from the ultrasound system, the renderer immediately displayed the volume to a conventional 19-inch cathode-ray tube monitor positioned in front of the surgeon. The high frame rate rendering allows for stereoscopic viewing via stereoscopic liquid crystal display shutter-glasses (eDimensional, West Palm, Fla). Left eye and right eye views are rendered from alternating the position and orientation of the volumetric data set and synchronized with the glasses shutter rate (Figure 1). By wearing the shutter-glasses, the surgeon uses the stereo-rendered 3DUS data for guiding a surgical procedure as he/she controls the surgical instruments.

\section{Study Design}

The experimental protocol was approved by the Children's Hospital Boston Institutional Animal Care and Use Committee. All animals received humane care in accordance with the 1996 Guide for the Care and Use of Laboratory Animals, recommended by the US National Institute of Health.

Six Yorkshire pigs weighing 70 to $80 \mathrm{~kg}$ were anesthetized by intramuscular injection of tiletamine/zolazepam $(7 \mathrm{mg} / \mathrm{kg})$ and xylazine $(4 \mathrm{mg} / \mathrm{kg})$ and intubated with a cuffed endotracheal tube and ventilated with a pressure control ventilator (Healthdyne 105; Healthdyne Technologies, Marietta, Ga). Anesthesia was maintained with $2 \%$ isoflurane. A median sternotomy was performed; a few stay sutures were placed on the pericardium to optimize access to the right atrium. The ultrasound transducer was inserted into a sleeve (CIVCO Medical Instruments, Kalona, Ia) filled out with an ultrasound gel (Parker Laboratories, Inc, Fairfield, NJ) providing approximately $2 \mathrm{~cm}$ of stand-off. The outer surface of the sleeve was watered with $0.9 \%$ sodium chloride solution and applied to the surface of the right atrium. Two purse-string sutures of 3-0 polypropylene were placed on the right atrial appendage for instrument insertion. After heparin was intravenously administered (100 U/ $\mathrm{kg}$ ), an ASD was created solely under RT3DE guidance as previously described. ${ }^{1,2}$ First, a transseptal puncture was performed, and a balloon catheter was inserted across the septum. After balloon atrial septostomy, the defect was enlarged with a Kerrison bone punch. Then, the defect was closed using an originally designed catheter-based patch delivery system, as previously described. ${ }^{2}$ The patch was attached around the defect by Nitinol mini-anchors deployed with an anchor delivery device under RT3DE control (Figure 2). For these experiments, the frame of the patch delivery device was left inside the heart as a reference point for a measurement of the accuracy of anchor placement.

RT3DE with SV display (group 1) was compared with RT3DE with standard 2D display (group 2) for guidance of ASD closure. Task performance measures for each anchor placement were completion time, trajectory of the tip of the anchor deployment device, and accuracy of the anchor placement. The starting point for the completion time and the trajectory was the moment when the surgeon first noticed the tip of the device on the echocardiography display (Figure 2). The trajectories were measured with electromagnetic tracking beads (Flock of Birds; Ascension Technologies, Burlington, Vt) as previously described. ${ }^{5}$ The tracker was fixed to the handle of the anchor deployment device. The ideal trajectory is a straight line from the starting point to the target. Differences between the instrument trajectory and a straight line are quantified using equation 3 : 


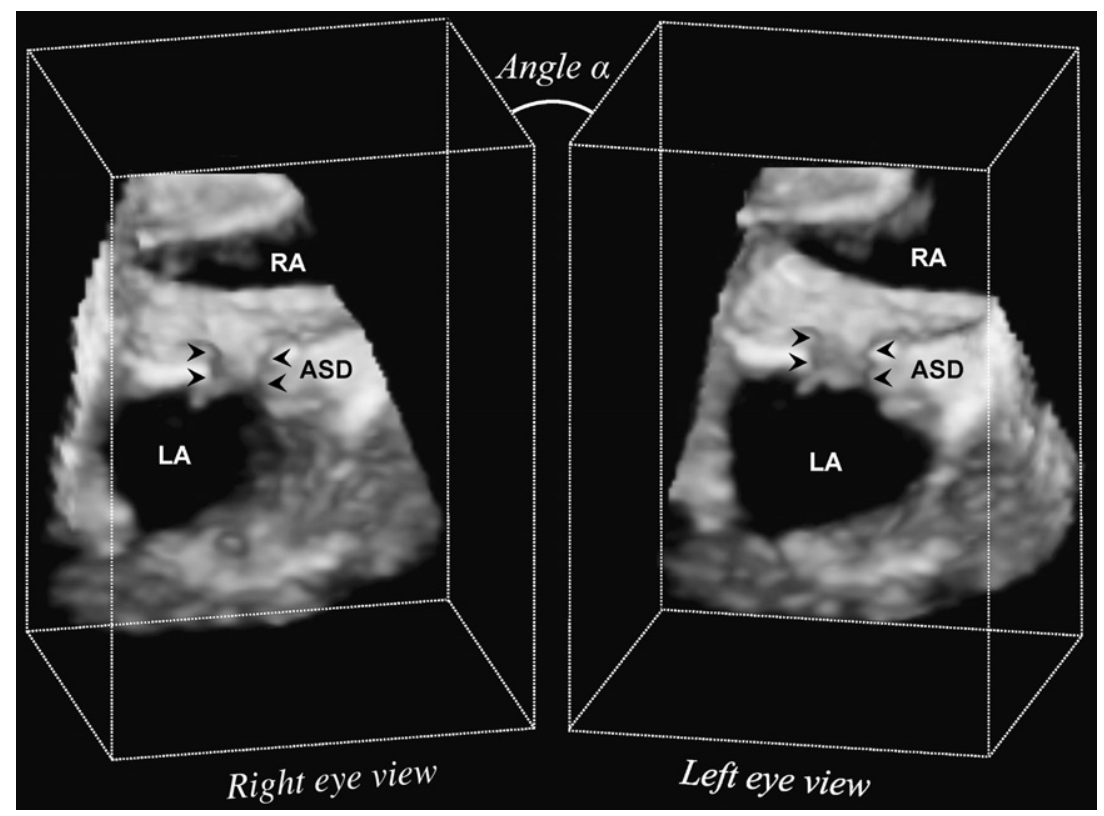

Figure 1. The volumetric data set of the created ASD (arrowheads) is sampled using parallel projection. Rays are cast simultaneously in a front-to-back fashion through the 3DUS data. Left eye and right eye views are separately generated by rendering the 3DUS volume from 2 viewpoints skewed by angle $\alpha$. $L A$, Left atrium; $R A$, right atrium.

\section{Statistical Analysis}

Analysis of the time required for complete anchor deployment, the tool-tip trajectory deviation, and the accuracy of each anchor placement was performed with the Student $t$ test using Matlab (Version R2006B, MathWorks, Natick, Mass).

\section{Disclosures and Freedom of Investigation}

The equipment and technology used in the study were purchased using academic funds. The authors had full control of the design of the study, methods used, outcome measurements, analysis of data, and production of the written report.

\section{Results}

\section{Atrial Septal Defect Creation}

The ASDs in both groups were created solely under RT3DE guidance with a standard 2D display. The mean ASD diameter measured by 2D color Doppler echocardiography jet was not significantly different for the SV display group $(6.1 \pm 1.0$ $\mathrm{mm}$; range 5.4-7.3 $\mathrm{mm}$ ) compared with the standard display group $(6.2 \pm 0.7 \mathrm{~mm}$; range 5.5-6.2 $\mathrm{mm})(P=.9)$.

\section{Atrial Septal Defect Closure}

An equal amount of the anchors $(\mathrm{N}=32)$ was deployed in each group. We used the patch with the same diameter

$(15 \mathrm{~mm})$ for all ASD closures in both groups. There were significant differences in speed and precision of instrument navigation between the 2 groups. The mean time of the anchor deployment for the SV display group was shorter by $44 \%$ compared with the standard display group: $9.7 \pm 0.9$ seconds versus $17.2 \pm 0.9$ seconds $(P<.001)$ (Figure 3,A). Anchor deployment device-tip trajectory tracking demonstrated greater navigational accuracy measured by means of trajectory deviation analysis. With SV RT3DE guidance, trajectory deviation decreased from $6.1 \pm 0.3 \mathrm{~mm}$ to $3.8 \pm 0.7 \mathrm{~mm}$, a 38\% improvement $(P<.01)$ (Figure 3, B). Typical paths for task completion are presented in Figure 4. Accuracy of anchor placement was not significantly different: $2.3 \pm 0.3 \mathrm{~mm}$ for the SV display group versus $2.3 \pm 0.3 \mathrm{~mm}$ for the standard display group (Figure 3,C). Sample postmortem photographs from animals are demonstrated in Figure 5.

\section{Discussion}

We observed that a custom-built real-time stereoscopic display of 3DUS images of the intracardiac structures significantly improves the surgeon's ability to navigate an instrument inside the beating heart. Stereoscopic display of 3D images improved the time of task completion and minimized deviation from an ideal trajectory, although accuracy of anchor placement was not improved.

To understand these findings, it is important to view the process of image-guided patch fixation as having 2 steps. The first step is advancement of the instrument from the insertion point at the right atrial free wall toward the target, the ASD patch. In this step, the surgeon relies on visual information to identify and track the surgical instrument and the cardiac structures within the field of view. For this task, the SV 

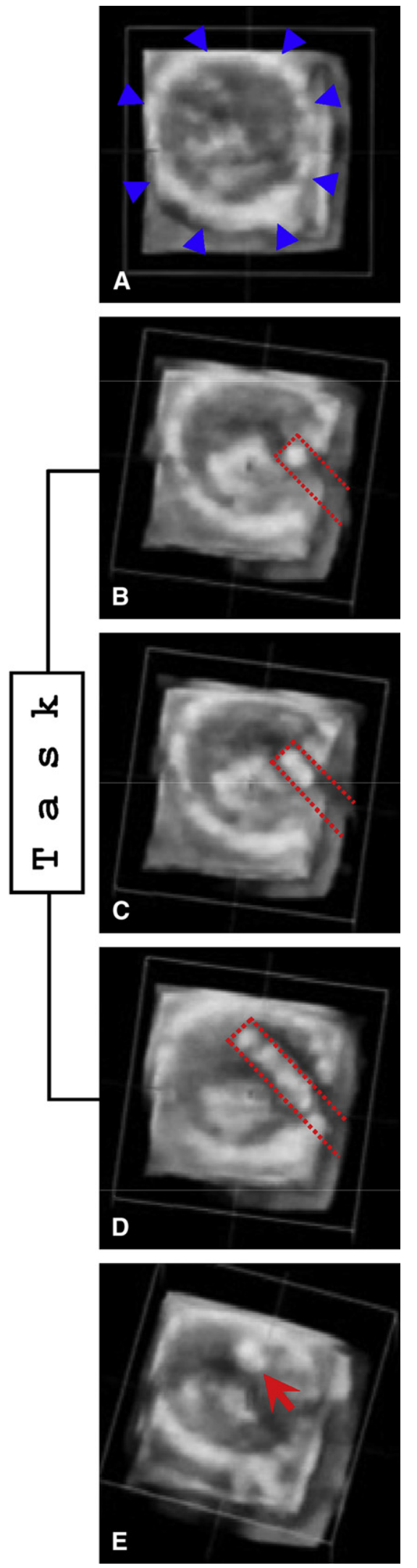

display provides a notable advantage over a conventional 2D display in the ability to navigate the instrument precisely, rapidly, and safely (Figures 3 and 4). The second step is patch attachment by deploying the anchor through the patch and underlying tissue. In this second step, once contact between the anchor deployment instrument and the patch material has been established, the operator performs fine positioning of the tool tip on the patch and deploys the anchor. For the second task, the surgeon relies less on visual information provided by the ultrasound image and considerably more on the tactile feedback from the contact with the patch polyester and the frame of the patch deployment device. The extent of operator experience with the procedure plays a significant role in accuracy of anchor placement. In our series, all the experiments were done by an operator who had significant experience with beating-heart intracardiac 3D echocardiography (3DE)-guided procedures. This may explain why there was no significant advantage of SV display in accuracy of anchor placement when compared with the 2D display. Because the first step of the procedure was done by the same operator, it is important to recognize that the SV display improved the speed of task performance and deviation from ideal instrument trajectory even when the operator had significant experience with the procedure. Subjectively, in all the SV RT3DE experiments the surgeon experienced greater confidence in instrument manipulation inside the beating heart using SV RT3DE for navigation.

In 3DUS diagnostic imaging, investigators first attempted to use the benefit of SV displays a decade ago. ${ }^{6-8}$ The technology in ultrasound imaging has made significant progress since that time. The ease of data acquisition, real-time 3D rendering, ability to focus on a specific anatomic structure, and a variety of additional quantification tools have enabled virtually routine application of 3DUS in cardiology practice. ${ }^{9}$ However, stereoscopic viewing of the 3DUS data has not been widely accepted. This can be partially explained by the absence of commercially available and easy to use stereoscopic visualization tools. In addition, experienced echocardiographers are able to diagnose most of the lesions using currently available 2D ultrasound and 3DUS techniques, although no studies have been performed comparing diagnostic abilities of the subjects using advanced SV versus conventional displays.

SV display in image-guided minimally invasive surgical interventions was first introduced in the early 1990s. ${ }^{10,11} \mathrm{Sev}-$ eral studies compared surgical performance in optical

Figure 2. Sequence of RT3DE images illustrating ASD patch closure. A, Self-expanding Nitinol frame (blue arrowheads) with the polyester patch is deployed and covers the ASD. B-D, The surgical task is demonstrated. The anchor deployment device (red dashed line) is advanced toward the target spot on the patch, and the Nitinol anchor is deployed attaching the patch to the septum. E, Final view of the deployed anchor (red arrow). 

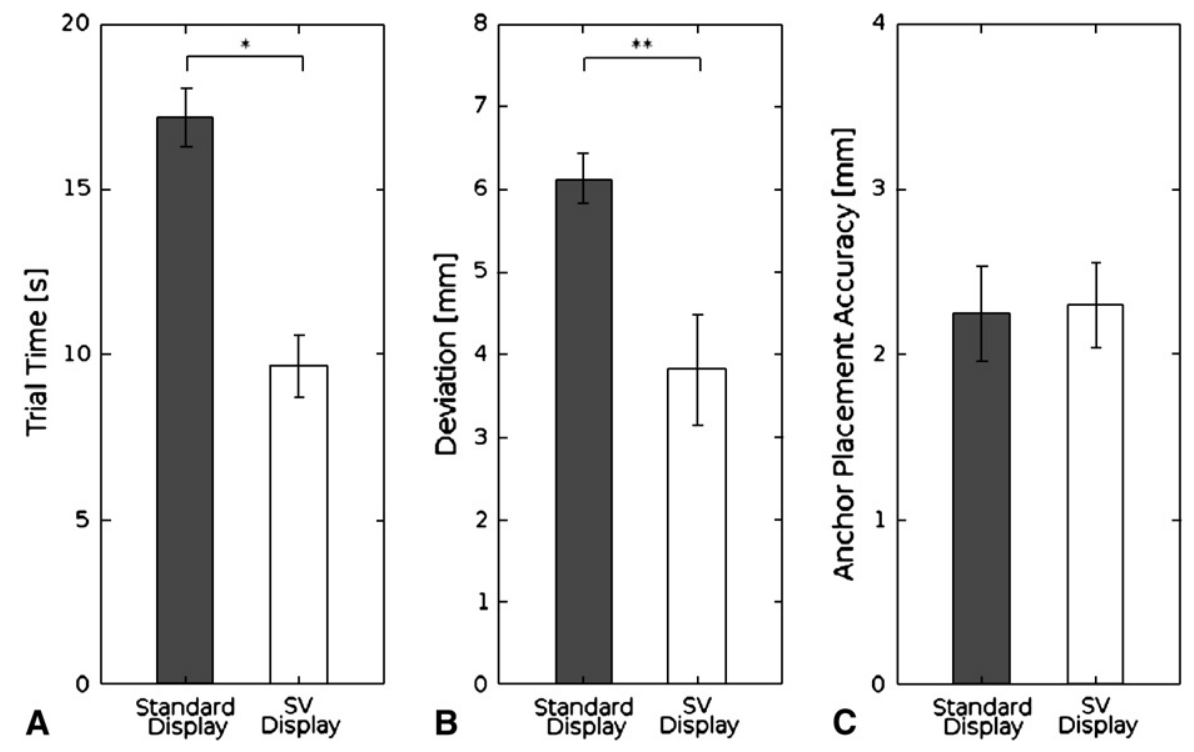

Figure 3. Task completion times (A), mean anchor deployment device tip trajectory deviations (B), and anchor placement accuracy (C). ${ }^{*} P<.001$, ${ }^{* *} \boldsymbol{P}<.01$. Error bars indicate standard error.

endoscopy-guided procedures using various SV technologies versus standard 2D displays, both in a laboratory and clinical setting. ${ }^{12-20}$ Some of the investigators suggested that the use of SV displays in endoscopic imaging had minor or no advantage for experienced laparoscopic surgeons but had a remarkable benefit for novices, ${ }^{13-16}$ whereas others did not find a significant difference. ${ }^{17-20}$ With the improvements in imaging technologies and introduction of high-definition display systems, investigators did not find a notable advantage in SV systems compared with a 2D high-definition display presentation. ${ }^{21}$ When a high-definition optical image is projected on a $2 \mathrm{D}$ screen, the operators are able to effectively use positional cues and rely on their previous experience to navigate the tip of an instrument and accurately manipulate the tissue. However, when SV was merged with high-resolution displays, as in the da Vinci telemanipulation system (Intuitive Surgical, Mountain View, Calif), an advantage of SV imaging was demonstrated in robotically assisted surgical procedures. Several reports have described that operators benefited from receiving additional depth information while manipulating in a limited space and relying solely on visual information with no haptic feedback. ${ }^{22,23}$

For control and navigation of surgical instruments to repair defects inside the beating heart, precise volumetric (3D) real-time imaging is required, because surgeons must recognize and manipulate delicate cardiac tissues within a rapidly moving and geometrically complex structure. In endoscopic procedures, surgeons traditionally are trained to base their judgments as to instrument navigation and tissue manipulation primarily on direct vision via optical endoscopic imaging. However, ultrasound imaging does not have the spatial resolution of optical imaging, and therefore the ability of the surgeon to identify surgical instruments and instrument position with respect to the target tissue is more limited. Although spatial resolution of current 3DUS systems has improved significantly when compared with systems available only a few years ago, the lack of fine detail makes interpretation of the depth of field difficult. The usual cues used by endoscopic surgeons to provide positional information of instruments within the field of view are not readily available with 3DUS imaging. We therefore hypothesized that stereoscopic displays would provide significantly better spatial information and depth perception to the surgeon compared with conventional 2D displays, even if the latter used high-definition cathode-ray tubes. Our findings confirm our hypothesis, even for an experienced endoscopic surgery operator.

Alternative imaging techniques for visualization inside the beating heart in real time have been described, including video-assisted cardioscopy using visible wavelength light. ${ }^{2,24}$ Although video-assisted cardioscopy offers detailed, highmagnification pictures of the target and provides greater confidence for fine instrument manipulations, depth of field is extremely limited and the scope window must be pressed directly against the target structures for visualization. ${ }^{2}$ Fiber optic infrared endoscopy was recently introduced to overcome the depth of field problem, because the wavelength used permits transmission through blood for a few millimeters. ${ }^{25}$ The depth of field, however, is still less than 1 to $2 \mathrm{~cm}$, making navigation through adult-sized cardiac structures difficult, requiring the use of other imaging techniques (eg, fluoroscopy). An additional limitation of current infrared systems is a relatively low frame rate, which requires significant computer processing for real-time imaging. Unlike intracardiac optical or infrared imaging, the ultrasound-based systems provide an opportunity to visualize a considerable volume of cardiac blood and tissue, which the optical imaging techniques cannot penetrate. 

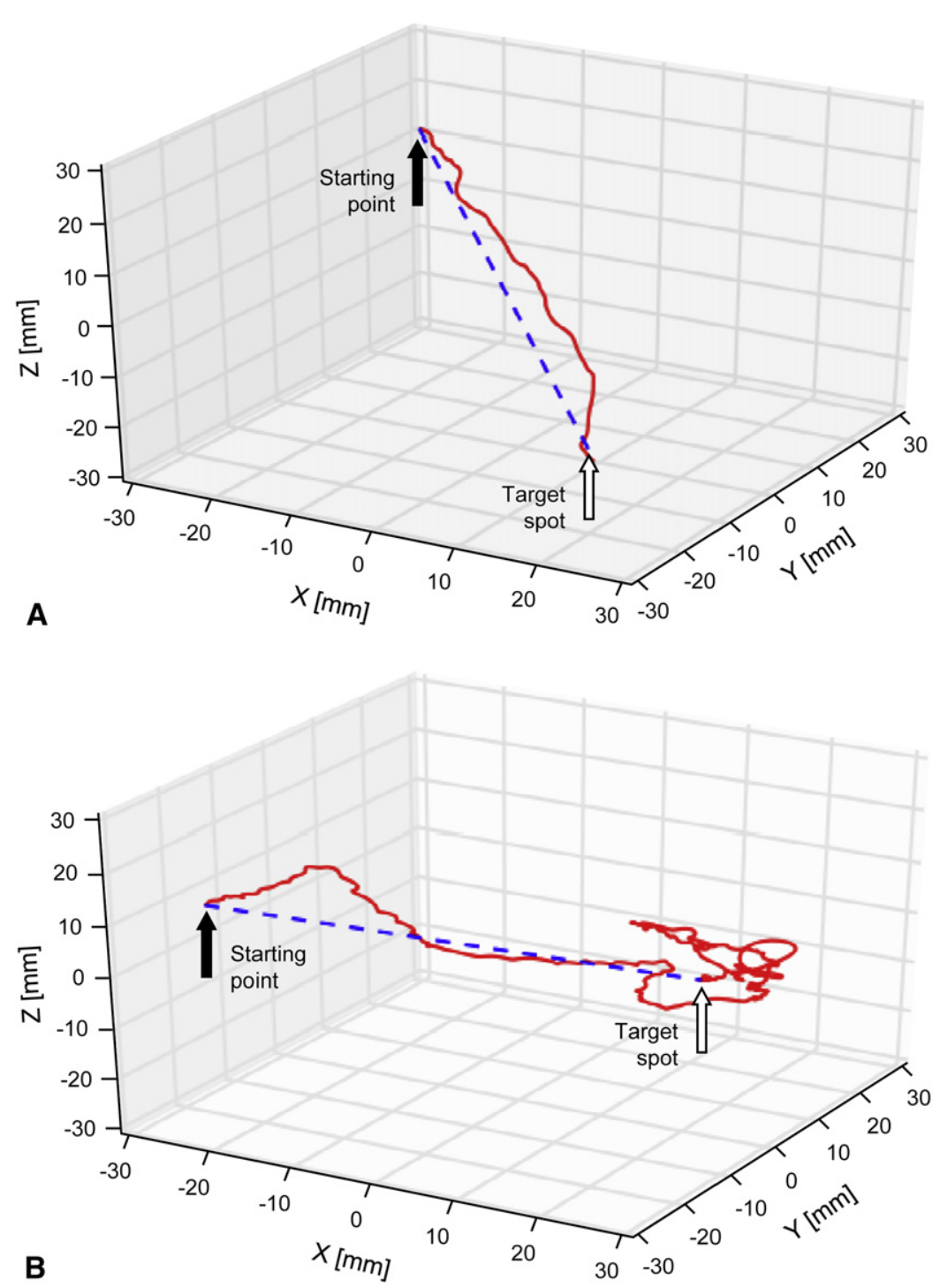

Figure 4. Typical paths for task completion using SV display (A) and standard display (B). The solid red line is the graphic representation of the anchor deployment device tip path from the starting point (black arrow) to the target spot (white arrow). The dashed blue line is an optimal trajectory.

\section{Study Limitations}

The experiments were done by a single operator with significant experience in endoscopic surgery and image-guided beating-heart surgery. Therefore, we were not able to compare the effect of SV 3DE on this task performance between individuals with various levels of surgical experience. However, our group previously reported the results of the performance evaluation study with an in vitro task in an ultrasound tank where the same stereo-rendering algorithm described above was used. ${ }^{26}$ Sixteen subjects (3 groups) with various experiences in endoscopic surgery were asked to perform in vitro surgical tasks with the surgical robot (Intuitive Surgical). Tasks error rates decreased by $50 \%$ with an SV display across all the groups, and all subjects completed tasks $28 \%$ faster with the stereo-display 3DUS compared with standard-display 3DUS, which corresponds to the results of the present study.

\section{Clinical Applications}

Recent reports of new image-guided beating-heart interventions, including transapical aortic valve and periventricular pulmonary valve implantation, ${ }^{27,28}$ mitral valvuloplasty, ${ }^{29}$ and septal defects closure, ${ }^{30}$ demonstrate increasing interest by the surgical community in such procedures and technologies. With the improved image quality of 3DUS, the complementary use of SV display technology would allow operators to precisely navigate various tools inside the beating heart for repair while minimizing trauma to neighboring structures. This, together with the development of new tools for such interventions, would enable the closure of complex septal defects, the removal of extra tissue inside the outflow tracts, and the potential repair of delicate, rapidly moving structures, such as mitral or aortic valve leaflets in the beating heart. 

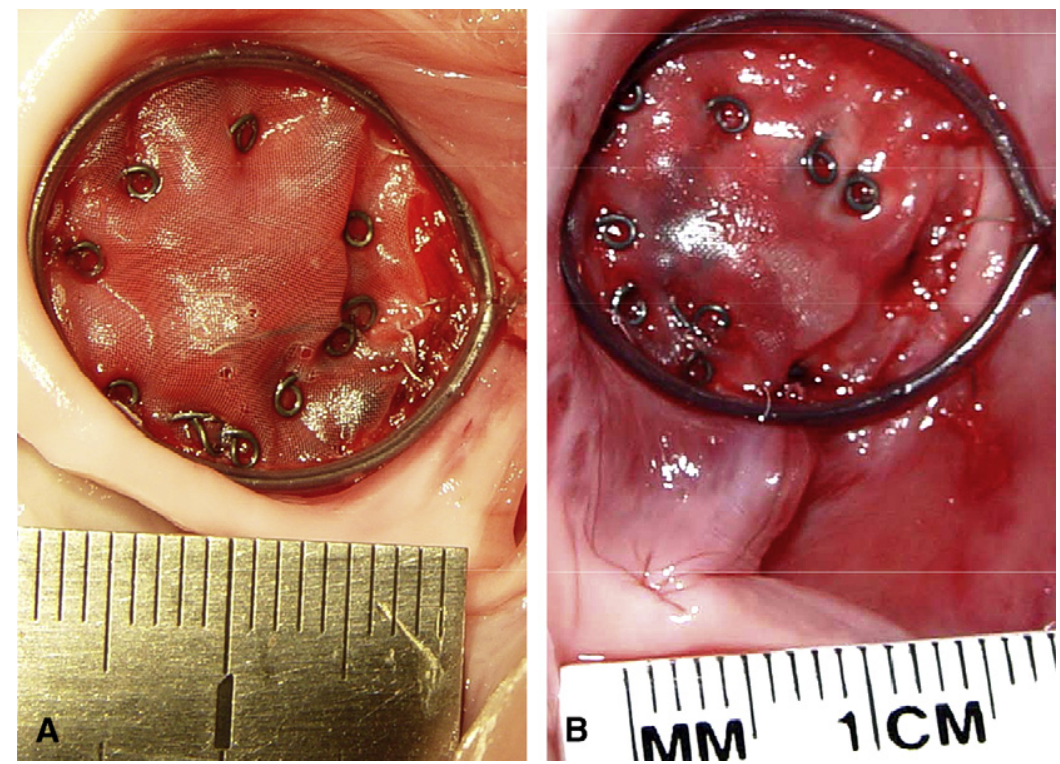

Figure 5. Sample postmortem photographs of the deployed patch and the anchors. SV display (A) and standard display (B).

\section{Conclusions}

Our study demonstrates that SV 3DE technology has significant advantages over the conventional display when used to guide beating-heart intracardiac surgical interventions. SV display combined with $3 \mathrm{DE}$ improved the visualization of 3DUS images, decreased the time required for surgical task completion, and increased the precision of instrument navigation, potentially improving procedure safety.

\section{References}

1. Suematsu Y, Martinez JF, Wolf BK, Marx GR, Stoll JA, DuPont PE, et al. Three-dimensional echo-guided beating heart surgery without cardiopulmonary bypass: atrial septal defect closure in a swine model. J Thorac Cardiovasc Surg. 2005;130:1348-57.

2. Vasilyev NV, Martinez JF, Freudenthal FP, Suematsu Y, Marx GR, del Nido PJ. Three-dimensional echo and videocardioscopy-guided atrial septal defect closure. Ann Thorac Surg. 2006;82:1322-6.

3. Kruger J, Westermann R. Acceleration techniques for GPU-based volume rendering. IEEE Visualization. 2003;287-92.

4. Novotny PM, Stoll JA, Vasilyev NV, del Nido PJ, Dupont PE, Howe RD. GPU based real-time instrument tracking with three dimensional ultrasound. Med Image Comput Comput Assist Interv. 2006; 9(Pt 1):58-65.

5. Cannon JW, Stoll JA, Salgo IS, Knowles HB, Howe RD, DuPont PE, et al. Real-time three-dimensional ultrasound for guiding surgical tasks. Comput Aided Surg. 2003;8:82-90.

6. Nelson TR, Pretorius DH. Visualization of the fetal thoracic skeleton with three-dimensional sonography: a preliminary report. AJR Am J Roentgenol. 1995;164:1485-8.

7. Riccabona M, Pretorius DH, Nelson TR, Johnson D, Budorick NE. Three-dimensional ultrasound: display modalities in obstetrics. J Clin Ultrasound. 1997;25:157-67.

8. Hernandez A, Basset O, Bremond A, Magnin IE. Stereoscopic visualization of three-dimensional ultrasonic data applied to breast tumours. Eur J Ultrasound. 1998;8:51-65.

9. Lang RM, Mor-Avi V, Sugeng L, Nieman PS, Sahn DJ. Three-dimensional echocardiography: the benefits of the additional dimension. J Am Coll Cardiol. 2006;48:2053-69.
10. Cuschieri A. Minimal access surgery and the future of interventional laparoscopy. Am J Surg. 1991;161:404-7.

11. Satava RM. 3-D vision technology applied to advanced minimally invasive surgery systems. Surg Endosc. 1993;7:429-31.

12. Durrani AF, Preminger GM. Three-dimensional video imaging for endoscopic surgery. Comput Biol Med. 1995;25:237-47.

13. Hofmeister J, Frank TG, Cuschieri A, Wade NJ. Perceptual aspects of two-dimensional and stereoscopic display techniques in endoscopic surgery: review and current problems. Semin Laparosc Surg. 2001;8:12-24.

14. Mueller-Richter UD, Limberger A, Weber P, Ruprecht KW, Spitzer W, Schilling M. Possibilities and limitations of current stereo-endoscopy. Surg Endosc. 2004;18:942-7.

15. Peitgen K, Walz MV, Holtmann G, Eigler FW. A prospective randomized experimental evaluation of three-dimensional imaging in laparoscopy. Gastrointest Endosc. 1996;44:262-7.

16. van Bergen P, Kunert W, Bessell J, Buess GF. Comparative study of two-dimensional and three-dimensional vision systems for minimally invasive surgery. Surg Endosc. 1998;12:948-54.

17. Taffinder N, Smith SG, Huber J, Russell RC, Darzi A. The effect of a second-generation 3D endoscope on the laparoscopic precision of novices and experienced surgeons. Surg Endosc. 1999;13:1087-92.

18. Chan AC, Chung SC, Yim AP, Lau JY, Ng EK, Li AK. Comparison of two-dimensional vs three-dimensional camera systems in laparoscopic surgery. Surg Endosc. 1997;11:438-40.

19. Hanna GB, Shimi SM, Cuschieri A. Randomised study of influence of two-dimensional versus three-dimensional imaging on performance of laparoscopic cholecystectomy. Lancet. 1998;351:248-51.

20. Mueller MD, Camartin C, Dreher E, Hanggi W. Three-dimensional laparoscopy. Gadget or progress? A randomized trial on the efficacy of three-dimensional laparoscopy. Surg Endosc. 1999;13:469-72.

21. van Bergen P, Kunert W, Buess GF. The effect of high-definition imaging on surgical task efficiency in minimally invasive surgery: an experimental comparison between three-dimensional imaging and direct vision through a stereoscopic TEM rectoscope. Surg Endosc. 2000;14: $71-4$.

22. Falk V, Mintz D, Grunenfelder J, Fann JI, Burdon TA. Influence of three-dimensional vision on surgical telemanipulator performance. Surg Endosc. 2001;15:1282-8.

23. Badani KK, Bhandari A, Tewari A, Menon M. Comparison of twodimensional and three-dimensional suturing: is there a difference in a robotic surgery setting? J Endourol. 2005;19:1212-5. 
24. Sogawa M, Moro H, Tsuchida M, Shinonaga M, Ohzeki H, Hayashi J. Development of an endocardioscope for repair of an atrial septal defect in the beating heart. ASAIO J. 1999;45:90-3.

25. Nazarian S, Knight BP, Dickfeld TL, Zviman MM, Jayanti VB, Amundson D, et al. Direct visualization of coronary sinus ostium and branches with a flexible steerable fiberoptic infrared endoscope. Heart Rhythm. 2005;2:844-8.

26. Novotny PM, Jacobsen SK, Vasilyev NV, Kettler DT, Salgo IS, DuPont PE, et al. 3D ultrasound in robotic surgery: a study of performance with stereo displays. Int J Med Robotics Comput Assist Surg. 2006;2:279-85.

27. Lichtenstein SV, Cheung A, Ye J, Thompson CR, Carere RG, Pasupati S, et al. Transapical transcatheter aortic valve implantation in humans: initial clinical experience. Circulation. 2006;114:591-6.
28. Schreiber C, Horer J, Vogt M, Fratz S, Kunze M, Galm C, et al. A new treatment option for pulmonary valvar insufficiency: first experiences with implantation of a self-expanding stented valve without use of cardiopulmonary bypass. Eur J Cardiothorac Surg. 2007; 31:26-30.

29. Feldman T, Wasserman HS, Herrmann HC, Gray W, Block PC, Whitlow $\mathrm{P}$, et al. Percutaneous mitral valve repair using the edge-toedge technique: six-month results of the EVEREST Phase I Clinical Trial. J Am Coll Cardiol. 2005;46:2134-40.

30. Bacha EA, Cao QL, Starr JP, Waight D, Ebeid MR, Hijazi ZM. Periventricular device closure of muscular ventricular septal defects on the beating heart: technique and results. J Thorac Cardiovasc Surg. 2003;126: $1718-23$.

\section{ON THE MOVE?}

\section{Send us your new address at least six weeks ahead}

Don't miss a single issue of the journal! To ensure prompt service when you change your address, please photocopy and complete the form below.

Please send your change of address notification at least six weeks before your move to ensure continued service. We regret we cannot guarantee replacement of issues missed due to late notification.

\section{JOURNAL TITLE:}

Fill in the title of the journal here.

\section{OLD ADDRESS:}

Affix the address label from a recent issue of the journal here.

\section{NEW ADDRESS:}

Clearly print your new address here.

Name

Address

City/State/ZIP

\section{COPY AND MAIL THIS FORM TO:}

Elsevier Inc.

Subscription Customer Service

6277 Sea Harbor Dr

Orlando, FL 32887
OR FAX TO:

407-363-9661

OR E-mail:

elspcs@elsevier.com
OR PHONE:

800-654-2452

Outside the U.S., call

407-345-4000 